\title{
The "noncausal causality" of quantum information
}

\author{
Vasil Penchev, vasildinev@gmail.com \\ Bulgarian Academy of Sciences: Institute of Philosophy and Sociology: \\ Dept. of Philosophy of Science
}

\begin{abstract}
The paper is concentrated on the special changes of the conception of causality from quantum mechanics to quantum information meaning as a background the revolution implemented by the former to classical physics and science after Max Born's probabilistic reinterpretation of wave function. Those changes can be enumerated so: (1) quantum information describes the general case of the relation of two wave functions, and particularly, the causal amendment of a single one; (2) it keeps the physical description to be causal by the conservation of quantum information and in accordance with Born's interpretation; (3) it introduces inverse causality, "backwards in time", observable "forwards in time" as the fundamentally random probability density distribution of all possible measurements of any physical quantity in quantum mechanics; (4) it involves a kind of "bidirectional causality" unifying (4.1) the classical determinism of cause and effect, (4.2) the probabilistic causality of quantum mechanics, and (4.3) the reversibility of any coherent state; (5) it identifies determinism with the function successor in Peano arithmetic, and its proper generalized causality with the information function successor in Hilbert arithmetic.

Key words: Hilbert arithmetic, probabilistic interpretation of quantum mechanics; quantum information, quantum-information conservation, qubit Hilbert space, reverse causality
\end{abstract}

\section{BACKGROUND: THE REVOLUTION OF QUANTUM CAUSALITY}

The concept of causality is fundamental for science constituting it in opposition to religion and often, even to common sense. Any observable phenomenon has its cause though it can be hidden. Science legitimates its existence also due to finding those hidden causes.

At first glance, quantum mechanics deprived physics of causality. This reason may explain the stubborn resistance and non-acceptance of quantum mechanics during decades even by great scientists such as Einstein.

The elucidation followed Max Born's interpretation, after which wave function could be interpreted as the characteristic function of a certain probability density distribution of any quantity in quantum mechanics and substituted the unambiguously determined single value of physical quantity featured in classical mechanics and physics. That replacement is fatal to causality as determinism, for which Einstein coined the metaphor of "God playing dice" (Einstein 1926) rejecting it.

However, Max Born himself pointed out that the change of wave function is rigorously deterministic though the result of a single measurement is fundamentally random (as that of any finite set of measurements, what all experiments are). This corresponds to the holism of quantum mechanics conserving determinism to the whole (of all possible experiments), but not to any finite subset of them. 
Furthermore, the discussion about causality in quantum mechanics was rather abstract and metaphysical corresponding to the mentality of the "founding fathers" of quantum mechanics, but not to that of their inheritors, whose slogan became "Shut up and calculate!" (Mermin 2004; Kaiser 2014). So, the problem about quantum causality was delivered to the philosophers of science, who in turn abandoned it gradually and left to sink into oblivion.

\section{HOW QUANTUM INFORMATION DESCRIBES QUANTUM CAUSALITY}

The specific subject of the paper is the interpretation and elucidation of quantum causality, implemented by theory of quantum information implicitly in virtue of its interpretation of quantum mechanics, merging it with information by its generalization to quantum information.

Indeed and in fact, "qubit" is defined as an "atom" (literally, "inseparable" in Greek) or a "quantum" (also metaphorically) of quantum causality: $\alpha \perp_{0}+\beta \perp_{1}$ where $\left.\alpha, \beta \in C:|\alpha|^{2}+|\beta|^{2}=1 ; \perp_{0}, \perp_{1} \subseteq \mathbb{H}\right):\left(\perp_{0}\right) \perp\left(\perp_{1}\right)$. That is: the superposition of two orthogonal subspaces (not necessarily to be convex to each other) normed unitarily and unambiguously by two complex numbers. Then, $\alpha \perp_{0}, \beta \perp_{1}$ can be interpreted correspondingly as "cause" and "effect", at that normed to their joint physical action: i.e. interaction.

The commutativity of the operation of addition "+" notates the reversibility of quantum causality. Furthermore, the mapping $[(\alpha, \beta) \rightarrow|\beta|] \subset[(C \otimes C) \rightarrow R]$ is a function but not a bijection, and thus, the reverse mapping $[(\alpha, \beta) \leftarrow|\beta|] \subset[(C \otimes C) \leftarrow R]$ is not a function: it is an ambiguous mapping so that more values than one of the mapping correspond to a certain value of its variable. The former mapping can be interpreted as the "normal" deterministic causality and "forwards in time", and the latter, as the specifically quantum, reverse causality and "backwards in time". Each of both as well as both together follow from the introduction of quantum information and its unit, a qubit.

The mapping $[(\alpha, \beta) \rightarrow|\beta|] \subset[(C \otimes C) \rightarrow R]$ can be interpreted also meaning that wave function after Born's interpretation is to be a characteristic function of a binary probability distribution of the two possible results $R_{0}, R_{1}$ of the measurement of a qubit and corresponding bijectively to $\perp_{0}, \perp_{1} \subseteq(\mathbb{H}$. Since the zero index is granted conventionally to "cause", and the unit index, to "effect", a qubit as unit of quantum causality represents the general case of the physical interaction of cause and effect unlike the particular case of determinism reducing quantum causality to the extreme particular case of causal (or temporal) irreversibility: cause acts physically upon effect (conventionally, $\beta=1$ ) while effect does not physically upon cause (following the same convention, $\alpha=0$ ).

If a qubit represents an elementary quantum state, e.g. the coherent state of the two possible spins of an electron, $|\alpha|^{2},|\beta|^{2}$ would notate the probabilities for either the one or the other values of the electron spin at issue to be measured following Born's interpretation and shared (but complemented) by the qubit interpretation of quantum information. 


\section{QUANTUM-INFORMATION CONSERVATION AND QUANTUM CAUSALITY}

Energy conservation in turn conserved in quantum mechanics in virtue of quantum conservation needs to be generalized to the latter (Penchev 2020 October 5) therefore forecasting nontemporal physical phenomena or interpreting all those of entanglement as the former furthermore sharing reverse causality. Thus, quantum information implying energy conservation implies also the "normal" deterministic causality "forwards in time", but along it, reverse causality featuring all phenomena of entanglement as well as even their inseparability in any coherent quantum state being reversible.

The total "sum" of all entanglement in the universe following from quantum-information conservation (Penchev 2020 October 5) can be projected anyway on the temporal screen of the "normal" deterministic causality where is admissible to be depicted only as "shadows" of matter and energy (Penchev 2021June 8); that is: as the experimentally corroborated "dark matter" and "dark energy", estimately about 20 times more than the visible ones.

In other words, the reverse causality of entanglement prevails crucially in the universe, and physical cognition supported only by energy conservation and respectively, by the "normal" deterministic causality forwards "in time" is hopelessly outdated.

The essential sense of quantum-information conservation as to quantum causality generalizing classical one consists in a new option (at that, very often met in the universe though not crucially necessary for human survival, however rather seemingly), namely: the transformation of the standard deterministic causality into reverse causality (the latter being representable as probability "veer of causes forwards in time") as well as vice versa by the necessary mediation of the coherent-state reversibility. Just the conservation of quantum information is able to manage that transformation unambiguously establishing how many (i.e. not "much") of classical causality (respectively, classical information) is to be equated to how much (i.e. not "many") of reverse causality (respectively, quantum information) ${ }^{1}$.

Since reverse causality can be identified as randomness or fundamental randomness, deterministic causality, as necessity, and the equation of both as actuality or reality, following the "sleng of philosophy", quantum-information conservation rules quantitatively the mutual transformation between randomness and necessity as reality.

\section{QUANTUM CAUSALITY AS INVERSE CAUSALITY}

Though, quantum causality (in a wider meaning as above) includes the usual deterministic causality of classical science as well its transformation into reverse causality, it (in a narrow meaning discussable now) means properly just the latter, reverse causality. Being directed "backwards in time", it is generalized by the fussy term or concept of randomness or

\footnotetext{
${ }^{1}$ The same equation can be realized as the "relativity of classical and quantum information, of bit and qubit" (Penchev 2021 April 12).
} 
fundamental randomness ${ }^{2}$ following the empirical time arrow of our experience "forwards in time".

Modern society believes that the influence of randomness can and must be restricted more and more, not being subject to human action and thus uncontrollable and unmanageable: threat for humankind. That diminishing of randomness is partly real (for the cognition of new and new variables influencing "flipping a coin" and due to science), partly seeming (for no cognition of any variables can resolve the problem about the state of "Schrödinger's cat").

The former and latter can be considered also as underlying the opposition of science and religion. Indeed religion of any kind all over the world declares hidden, divine reality to which the religion at issue is relevant (or even, the only relevant one). On the contrary, science (at least until now) is devoted to the visible, profane reality of empirical and experimental experience. However, the divine (or sacral) reality of religion can be identified (interpretatively) as the sphere of fundamental randomness and thus obeying reverse causality (in detail in: Penchev 2021 April 12).

If that interpretation is correct or can be accepted to be valid under additional conditions, religion can be considered noy only "als strenge Wissenschaft" (what was Husserl's intention in relation to philosophy), but even as quantitative and experimental, investigating and transforming the mutual transformation between randomness and necessity as reality.

Of course, the belief, rites and rituals of religion would be useless and absolutely irrelevant to that research just as they are to any theory and experiments for gravitation (for example). The conflict of science and religion would continue (or even increase) probably, due to the redline of whether fundamental randomness is accessible to scientific study or on the contrary, this is the tabooed area of God's will.

\section{BIDIRECTIONAL CAUSALITY IN THREE HYPOSTASES}

The conclusion of the three hypostases of quantum causality being bidirectional, though eventually valid as to quantum phenomena, can met the following decisive objection and resistance to be applied to the macroscopic experiments of classical physics and science or to the immediate experience shared by all human beings (as the "invasion" of quantum mechanics into the sacral divine area of God's will suggests), namely:

Reverse causality is relevant only to the microscopic quantum phenomena where both Heisenberg uncertainty and Planck constant are essential and able to influence the observed experimental results considerably. However, it is absolutely inapplicable to the macroscopic human experience since the corresponding quantities of physical actions are much, much greater

\footnotetext{
${ }^{2}$ The term and concept of fundamental randomness distinguishes the randomness involved by quantum mechanics from the usual one of common sense. It can be visualized opposing "Schrödinger's cat" versus "flipping a coin" both sharing a space of events isomorphic to a bit of classical information ("dead/ alive" and "heads/ tails", accordingly). Nonetheless, the determinist prediction of the latter is possible meaning a finite set of relevant variables. On the contrary, this is impossible to the former according to the Kochen-Specker (1967) theorem: thus, guessing the state of "Schrödinger's cat" is a "not-P, but NP" problem (following the formulation of the "P vs NP problem"), but "flipping a coin is a "P and NP problem" (Penchev 2020 August 5).
} 
than the magnitude of the Planck constant. Speaking loosely, the size of matter and energy does matter: thus, only the microscopic quantum world is unstable under both uncertainty and reverse causality. Ergo, the world of our experience is inherently deterministic and predictable, on the one hand, or if one insists that randomness or God's will is essential for it, only religion, but not science, can relate to them, on the other hand.

A frequent response to that disagreement pays attention to the option for any quantum events to be amplified by dissipativity and thus to become noticeable macroscopically. In particular, this is the most common method of measurement in quantum mechanics. Then, any quantum phenomenon can call a fundamental random and unpredictable effect even crucial or fatal as after "Schrödinger's cat". Human brain - mind can be interpreted as such a dissipative amplifier or preamplifier of quantum randomness (or "God's Will" theologically), by which immaterial thoughts can cause physical actions after the neuron impulses be amplified additionally by the human body and its muscles.

Another and much more interesting kind of answer to the same objection explores the identification of the local space obeying quantum uncertainty with the global space of the apparatus and classical mechanics, which is due to the idempotency of the two dual Hilbert spaces of quantum mechanics or gauge symmetries meant by the Standard model. Then, the idempotency of both time arows (respectively, that of both deterministic and reverse causality) is interpreted to be isomorphic to the former as well as dependable on the choice of an either internal or external reference frame. Meaning their relativity investigated in another paper (Penchev 2021 April 2012) one can speak of quantum causality as relative, or invariant to deterministic and reverse causality.

Then, the "three hypostases" of quantum causality can be unified in virtue of the same relativity or invariance meant also by quantum-information conservation.

\section{HILBERT ARITHMETIC AND BIDIRECTIONAL CAUSALITY}

Deterministic causality can be represented mathematically as a well-ordering in time therefore implying the axiom of induction in arithmetic and both axiom of choice and well-ordering "theorem" in set theory. Though a well-ordering as in arithmetic as in set theory means the same structure isomorphic to that of natural numbers they are inconsistent to each other as the Gödel (1931) incompleteness theorems demonstrate. As far as they can be interpreted as the deterministic and quantum causality correspondingly, the latter two ones are inconsistent to each other just as the former two ones: deterministic causality is either incomplete or inconsistent to quantum causality, thus not being able to offer any complete description of reality.

On the contrary, the theorem of the absence of hidden variables in quantum mechanics (Neumann 1832; Kochen, Specker 1967) can be interpreted as the completeness of the mathematical formalism itself (namely that of the separable complex Hilbert space being able to be represented as the qubit Hilbert space, and thus, as "quanta" of quantum causality), and then, as the completeness of quantum causality (which is not true as to deterministic causality in virtue of the cited Gödel theorems). 
The completeness of quantum mechanics, as confirmed experimentally very well, can serve to build an arithmetic generalizing the standard Peano arithmetic, but unlike the latter, to be consistent with set theory. That generalization is Hilbert arithmetic just doubling Peano arithmetic by an anti-isometric twin therefore implying all the pairs of "number-sake" triples, the one of which belongs to the one counterpart of Peano arithmetic, the second one, to the other, twin and anti-isometric counterpart of Peano arithmetic, and the third one, to the coherent superposition of the former two ones, therefore deprived of any well-ordering. Each of those triple is isomorphic to a bit of classical information.

The structure of Hilbert arithmetic can be defined from the qubit Hilbert space as follows. A unit of Hilbert arithmetic is the class of equivalence of all possible values of a qubit, or in other words, an "empty" qubit of quantum information. Thus, Hilbert arithmetic is able to share the property of unitarity of the separable complex (or qubit) Hilbert space, which in turn underlies the completeness of both structures. Even they can be identified as the same under the additional condition that the qubits be defined as transfinite ordinal numbers, but less than the countable ordinal numbers and thus being the countable equivalent of inaccessible ordinals (respectively, inaccessible countable cardinals). The same ones can be granted definitively to be "transfinite natural numbers" implied by the complement of Peano arithmetic to Hilbert arithmetic following the way described above.

Hilbert arithmetic (being isomorphic or complementary to the qubit Hilbert space) is able to demonstrate all properties of quantum causality as well as its relation to deterministic causality properly and only mathematically. Its completeness together with the incompleteness of deterministic causality implies the existence of physical phenomena being inherently, or fundamentally random: at least, they should be at least the "half of the world". Particularly, "dark matter" and "dark energy" are to be enumerated among them.

\section{CONCLUSIONS}

The transition from quantum mechanics to quantum information reflects on a wider understanding of quantum causality, the features of which are enumerated sequentially section by section above. The unit of quantum information can be interpreted as a quantum of quantum causality: as a ratio (defined by the coefficients $\alpha, \beta$ ) of the interaction of deterministic and reverse causality. Quantum causality generalizes deterministic causality and relies on quantum-information conservation in turn generalizing energy conservation, to which deterministic causality is linked closely.

Particularly, the existence of nontemporal physical phenomena can be predicted and the very well corroborated experimentally "dark matter" and "dark energy" are to be enumerated among them.

Quantum causality can be defined in a narrow sense, as reverse causality, and then, three "hypostases" of quantum causality in a wide meaning being linked to irreversibility or reversibility of time: the standard deterministic causality irreversible and "forwards in time"; the proper quantum (or reverse) causality also irreversible, but "backwards in time"; at last the reversible coherent state sharing simultaneously both former irreversible ones. 
That bidirectional causality in three hypostases can be formalized as a fundamental mathematical structure and generalizing Peano arithmetic in a way to be consistent to set theory and therefore overcoming the restriction of the Gödel incompleteness. It can be called Hilbert arithmetic and can be identified either as complementary or as equivalent to the qubit Hilbert space in turn equivalent to the separable complex Hilbert space of quantum mechanics. Transfinite natural numbers can be defined in the framework of Hilbert arithmetic so that a bijection exists between all transfinite natural numbers and all elements (wave functions) of the separable complex Hilbert space.

\section{References:}

Born, M. (1949) Natural philosophy of cause and chance being the Waynflete lectures delivered in the college of St. Mary Magdalene 1948. Oxford: Oxford University Press.

Einstein, Albert (1926) "Letter to Max Born, 16 December 1926," in: Albert Einstein Max Born Briefwechsel 1916 - 1955 (kommentiert von Max Born). München: Nymphenburger Verlagshandlung, 1969: 129-130.

Gödel, K. (1931) "Über formal unentscheidbare Sätze "Principia mathematica" und verwandter Systeme I," Monatshefte für Mathematik 38 (1): 173-198.

Kaiser, D. (2014) "History: Shut up and calculate!" Nature 505 (7482) 153-155. https://doi.org/10.1038/505153a .

Kochen, S \& E. P. Specker (1967) "The problem of hidden variables in quantum mechanics," Journal of Mathematics and Mechanics 17 (1): 59 - 87 (1968).

Mermin, N. D. (2004) “Could Feynman Have Said This?” Physics Today 57 (5): 10-11. https://doi.org/10.1063/1.1768652.

Penchev, V. (2021 June 8) "The Symmetries of Quantum and Classical Information. The Resurrected 'Ether' of Quantum Information," SSRN, https://papers.ssrn.com/sol3/papers.cfm?abstract id=3861105.

Penchev, V. (2021 April 12) "Both classical \& quantum information; both bit \& qubit: transcendental time, or the phenomenon of time," SSRN, https://papers.ssrn.com/sol3/papers.cfm?abstract id=3823665.

Penchev, V. (2020 October 5) "Quantum-Information Conservation. The Problem About 'Hidden Variables', or the 'Conservation of Energy Conservation' in Quantum Mechanics: A Historical Lesson for Future Discoveries," SSRN, https://dx.doi.org/10.2139/ssrn.3675319 or https://papers.ssrn.com/sol3/papers.cfm?abstract id=3675319.

Penchev, V. (2020 August 5) "A Class of Examples Demonstrating that 'P $\neq$ NP' in the "P vs NP' Problem," SSRN, https://papers.ssrn.com/sol3/papers.cfm?abstract id=3647038 or https://dx.doi.org/10.2139/ssrn.3647038, 